\title{
Estimated 24-h urinary sodium and sodium-to-potassium ratio are predictors of kidney function decline in a population-based study
}

\author{
Deriaz, Denis ; Guessous, Idris ; Vollenweider, Peter ; Devuyst, Olivier ; Burnier, Michel ; Bochud, \\ Murielle ; Ponte, Belen
}

\begin{abstract}
BACKGROUND: The prevalence of chronic kidney disease (CKD) is increasing worldwide in part due to population ageing. Identifying risk factors for age-related kidney function decline could help in understanding mechanisms for kidney ageing. Sodium and potassium intakes are associated with CKD progression in the renal population, but little is known on their role in renal function decline [mean estimated glomerular filtration rate variation $(\Delta \mathrm{eGFR})]$ in the general adult population. METHOD: We therefore explored the association of urinary sodium and potassium excretions with $\Delta \mathrm{eGFR}$ in a longitudinal population-based cohort. We estimated 24-h urinary sodium (eUNa), potassium (eUK) and sodium-to-potassium ratio $(\mathrm{eUNa} / \mathrm{K})$ from spot urine using Kawasaki formulae. We performed multivariate linear regression models studying the association of eUNa, eUK and eUNa/K with yearly $\triangle$ eGFR, taking several covariates into account, including baseline eGFR and albuminuria. RESULTS: There were 4141 white participants from which $54.3 \%$ were women. Median age was 51.5 [43.6-60.6] years and mean baseline eGFR 88 (SD 15) $\mathrm{ml} / \mathrm{min}$ per $1.73 \mathrm{~m}$. During a median follow-up of 5.4 years, mean $\triangle \mathrm{eGFR}$ was $-0.59(\mathrm{SD} 1.68) \mathrm{ml} / \mathrm{min}$ per $1.73 \mathrm{~m}$ per year. In the fully adjusted model, high eUNa and $\mathrm{eUNa} / \mathrm{K}$ were associated with faster renal function decline with standardized coefficients $=-0.07(95 \%$ confidence interval: -0.11 to -0.04$)$ and $=-0.05$ (95\% confidence interval: -0.08 to -0.02$)$, respectively. By contrast, eUK, taken alone, showed no association. CONCLUSION: These results suggest that dietary sodium and potassium intakes may play a role in kidney function decline in the general adult population. Whether lowering sodium and increasing potassium in the diet may help in CKD prevention needs further exploration.
\end{abstract}

DOI: https://doi.org/10.1097/hjh.0000000000002098

Posted at the Zurich Open Repository and Archive, University of Zurich

ZORA URL: https://doi.org/10.5167/uzh-177054

Journal Article

Published Version

Originally published at:

Deriaz, Denis; Guessous, Idris; Vollenweider, Peter; Devuyst, Olivier; Burnier, Michel; Bochud, Murielle; Ponte, Belen (2019). Estimated 24-h urinary sodium and sodium-to-potassium ratio are predictors of kidney function decline in a population-based study. Journal of Hypertension, 37(9):1853-1860.

DOI: https://doi.org/10.1097/hjh.0000000000002098 


\title{
Original Article
}

\section{Estimated 24-h urinary sodium and sodium-to- potassium ratio are predictors of kidney function decline in a population-based study}

\author{
Denis Deriaz ${ }^{a}$, Idris Guessous ${ }^{a, b}$, Peter Vollenweider $^{c}$, Olivier Devuyst ${ }^{d}$, Michel Burnier ${ }^{\mathrm{e}}$, \\ Murielle Bochud $^{a, *}$, and Belen Ponte ${ }^{a, f, *}$
}

Background: The prevalence of chronic kidney disease (CKD) is increasing worldwide in part due to population ageing. Identifying risk factors for age-related kidney function decline could help in understanding mechanisms for kidney ageing. Sodium and potassium intakes are associated with CKD progression in the renal population, but little is known on their role in renal function decline [mean estimated glomerular filtration rate variation $(\Delta \mathrm{eGFR})]$ in the general adult population.

Method: We therefore explored the association of urinary sodium and potassium excretions with $\triangle \mathrm{eGFR}$ in a longitudinal population-based cohort. We estimated 24-h urinary sodium (eUNa), potassium (eUK) and sodium-topotassium ratio (eUNa/K) from spot urine using Kawasaki formulae. We performed multivariate linear regression models studying the association of eUNa, eUK and eUNa/K with yearly $\triangle \mathrm{eGFR}$, taking several covariates into account, including baseline eGFR and albuminuria.

Results: There were 4141 white participants from which 54.3\% were women. Median age was 51.5 [43.6-60.6] years and mean baseline eGFR 88 (SD 15) $\mathrm{ml} / \mathrm{min}$ per $1.73 \mathrm{~m}^{2}$. During a median follow-up of 5.4 years, mean $\Delta$ eGFR was -0.59 (SD 1.68) $\mathrm{ml} / \mathrm{min}$ per $1.73 \mathrm{~m}^{2}$ per year. In the fully adjusted model, high eUNa and eUNa/K were associated with faster renal function decline with standardized coefficients $\beta=-0.07$ (95\% confidence interval: -0.11 to -0.04$)$ and $\beta=-0.05$ (95\% confidence interval: -0.08 to -0.02$)$, respectively. By contrast, eUK, taken alone, showed no association.

Conclusion: These results suggest that dietary sodium and potassium intakes may play a role in kidney function decline in the general adult population. Whether lowering sodium and increasing potassium in the diet may help in CKD prevention needs further exploration.

Keywords: renal function decline, salt, sodium, urinary sodium-to-potassium ratio

Abbreviations: 25D, 25-hydroxyvitamin D; ACR, albuminto-creatinine ratio; CKD, chronic kidney disease; CoLaus, Cohort Lausanne study; DASH, Dietary Approaches to Stop Hypertension; eGFR, estimated glomerular filtration rate; eUK, estimated 24-h urinary potassium excretion; eUNa/K, eUNa divided by eUK; eUNa, estimated 24-h urinary sodium excretion; $\mathrm{Na} / \mathrm{K}$ ratio, sodium-to-potassium ratio;
NHANES, The National Health and Nutrition Examination Survey; PREVEND, The Prevention of Renal and Vascular End-Stage Disease study; UCr, urinary concentration of

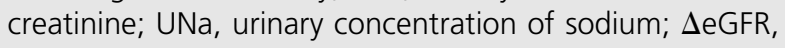
mean estimated glomerular filtration rate variation

\section{INTRODUCTION}

$\mathrm{H}$

igh-sodium intake and low-potassium intake are known dietary risk factors for arterial hypertension (AH) [1-3]. High-potassium intake reduces blood pressure (BP) and the risk of stroke [4]. Data regarding their role in cardiovascular morbidity and mortality are more controversial. In the US The National Health and Nutrition Examination Survey (NHANES) study, sodium intake and sodium-to-potassium intake ratio estimated by 24-h dietary recalls were associated with higher all-cause and cardiovascular mortality, whereas potassium intake appeared to be protective, with the strongest predictive value for the sodium-to-potassium intake ratio [5]. In the Prospective Urban Rural Epidemiology study, a large worldwide study, similar results were obtained using the Kawasaki formula to estimate 24-h urinary sodium and potassium excretions [3]. A similar association with cardiovascular events was found by using sodium-to-potassium excretion ratio in 24-h urine [6]. On the contrary, some studies rather showed an increase in mortality with lower sodium intake either at population or more recently at a community level $[7,8]$.

Journal of Hypertension 2019, 37:1853-1860

anstitute of Social and Preventive Medicine (IUMSP), Lausanne University Hospital (CHUV), Lausanne, 'Unit of Population Epidemiology, Division of Primary Care Medicine, Department of Community Medicine and Primary Care and Emergency Medicine, Geneva University Hospitals, Geneva, 'Department of General Internal Medicine, Lausanne University Hospital (CHUV), Lausanne, 'Institute of Physiology, University of Zurich, Zurich, 'Service of Nephrology and Hypertension, Lausanne University Hospital (CHUV), Lausanne and 'Division of Nephrology, Department of Medicine Specialities, Geneva University Hospitals (HUG), Geneva, Switzerland

Correspondence to Belen Ponte, Senior Lecturer, Division of Nephrology, Department of Medicine Specialties, University Hospital of Geneva, Ch Gabrielle-Perret-Gentil 4, 1211 Geneva, Switzerland. Tel: +41 0795533 245; e-mail: belen.ponte@hcuge.ch *Murielle Bochud and Belen Ponte are joint last authors.

Received 23 October 2018 Revised 7 February 2019 Accepted 3 March 2019 J Hypertens 37:1853-1860 Copyright (C) 2019 Wolters Kluwer Health, Inc. All rights reserved.

DOI:10.1097/HJH.0000000000002098 
A few observational studies explored the association of sodium intake with renal outcomes either by using 24-h urinary sodium excretion [9-13] or dietary recalls [14-16]. In most of them, high-sodium intake was associated with a higher risk of kidney function decline [15] and a more rapid progression to chronic kidney disease (CKD) [9], although an inverse association was found cross-sectionally in the NHANES study [16] and no association was found in the Prevention of Renal and Vascular End-Stage Disease (PREVEND) study [13]. A high-sodium intake can have an effect on kidney function through direct long-term BP effect on vessels, thereby accelerating arteriolosclerosis. High sodium and high BP can both induce hyperfiltration and glomerular damage in the long term. Other potential mechanisms are by modulating intra-renal transforming growth factor (TGF)- $\beta 1$ production, a fibrinogenic factor participating in CKD progression [17], and/or by inducing adenosine-related vasoconstriction in the macula densa.

Potassium intake seems to have a protective effect on renal outcomes [9,11-13,16,18-20]. Most observational studies analysed 24-h urinary potassium excretion $[9,11,12,19]$, one looked at serum potassium [18] and two considered self-reported diet $[16,20]$. Recently, the PREVEND study reported that a low 24-h urinary potassium excretion was rather associated with increased 10-year risk of CKD [13]. The mechanisms underlying the protective role of potassium on renal function are not clear, but could be related to decreased vascular stiffness and nitric oxide release and reduced BP sensitivity to sodium intake $[21,22]$.

A high urinary sodium-to-potassium ratio $(\mathrm{Na} / \mathrm{K}$ ratio $)$ jointly captures both high-sodium intake and low-potassium intake [23] and should therefore allow to identify a complex set of unhealthy dietary patterns, such as a diet rich in salty products and poor in fruits and vegetables. The practical usefulness of urinary $\mathrm{Na} / \mathrm{K}$ ratio for human studies is further enhanced by the availability of self-monitoring devices [24], by the facts that the ratio is less dependent on the urine collection or creatinine measurement and that the spot ratio better correlates to the 24-h ratio than sodium or potassium taken individually [25]. Sodium-to-potassium intake ratio may represent a better dietary biomarker to capture cardiorenal risk than either sodium or potassium intakes alone [23]. Jointly considering sodium and potassium intakes appears to be particularly relevant for the kidney-related pathogenesis of $\mathrm{AH}$ [26].

We explored the association of urinary sodium and potassium excretions as well as urinary $\mathrm{Na} / \mathrm{K}$ ratio, derived from morning spot urine, with age-related kidney function decline in a population-based cohort [Cohort Lausanne study (CoLaus)]. We hypothesized that high urinary sodium and $\mathrm{Na} / \mathrm{K}$ ratio would be predictive of a faster age-related kidney function decline and that high urinary potassium would rather be protective.

\section{METHODS}

\section{The Cohort Lausanne study}

One of the main goals of the CoLaus study was to determine the prevalence of cardiovascular risk factors and diseases in the general population. CoLaus is a longitudinal cohort study including a general population-based sample from the city of Lausanne in Switzerland. The baseline examination started in 2003-2006 with the first follow-up on average 5-year later (2009-2012). The complete sample method has already been described [27]. Briefly, a simple randomization from the city of Lausanne's register selected 19830 people, representing $35 \%$ of the registered population, who were then contacted by post. Inclusion criteria were written informed consent; aged from 35 to 75 years old; white origin. They were 6184 people responding to the inclusion criteria and who completed the baseline investigation, then $76 \%(n=4679)$ of these also completed the follow-up. The study respects the Declaration of Helsinki and was approved by the Ethics Committee of the Canton of Vaud.

\section{Biological and clinical data}

The participants came to the University Hospital from Lausanne for a clinical examination, after an overnight fast. BMI was defined as weight/height in $\mathrm{kg} / \mathrm{m}^{2}$. Waist circumference was measured following standardized recommendations [27]. Participants remained at rest in a seated position for at least $10 \mathrm{~min}$ and BP was measured three times using an Omron-automated oscillometric sphygmomanometer. The average of the last two measurements was used for analyses.

Venous blood samples were drawn during the clinical examination. During the same visit, one single spot urine sample was obtained from the second voiding urine specimen in the morning. All samples (blood and urine) were taken in the morning while the participants were still fasting. Venous blood glucose, lipid profile and renal function tests as well as urinary electrolytes were analysed by standard clinical methods. Serum 25 -hydroxyvitamin D (25D) was measured centrally by an ultra-high-pressure liquid chromatography-tandem mass spectrometry system. Month-specific tertiles were calculated for serum 25D because of seasonal concentration variations. Ultrasensitive C-reactive protein (CRP) was measured by immunoassay and high-sensitivity latex assay. Serum and urinary creatinine was measured using the Jaffe isotope dilution mass spectrometry (IDMS) traceable measurement kinetic compensated method (Roche Diagnostics, Switzerland; coefficient of variation (CV) 2.9-0.7\%). Albuminuria was measured using a bromocresol green assay (Roche Diagnostics, Switzerland; CV 2.5-0.4\%) and was expressed in $\mathrm{mg} / \mathrm{dl}$. Albumin-to-creatinine ratio (ACR) was calculated as the ratio of albumin over creatinine in $\mathrm{mg} / \mathrm{g}$. Urinary sodium and potassium were determined by indirect potentiometry (UniCEl DxC 800 Synchron System; Beckman Coulter, Brea, California, USA).

Participants had to complete a questionnaire about their socioeconomic status, cardiovascular risk factors and current medication. The level of education was classified as 'low', 'middle' or 'high'. Participants were considered physically 'active' if they completed at least 20 min of physical activity once per week. They were considered 'smokers' only if they were active smokers. Diabetes was defined as presence of current - or previous - antidiabetic treatment or a fasting glycemic value greater than $7 \mathrm{mmol} / \mathrm{l}$. Hypertension medication was reported by the patient. 


\section{Estimated glomerular filtration rate}

Estimated glomerular filtration rate (eGFR) was calculated from the baseline and 5-year follow-up serum creatinine using the Chronic Kidney Disease - Epidemiology Collaboration (CKD-EPI) equation [28]. Then, annual mean CKDEPI variation [mean eGFR variation $(\Delta e G F R)$ ] used two creatinine values, and was defined as:

$$
\Delta e G F R=\frac{\left[C K D-E P I_{f_{\text {ollow_up }}}-C K D-E P I_{\text {baseline }}\right]}{\left[\left(\text { day }_{\text {follow_up } \left.\left._{-}-\text {day }_{\text {baseline }}\right) / 365.25\right]} .\right.\right.}
$$

\section{Estimated 24-h urinary sodium excretion and estimated 24-h urinary potassium excretion}

We estimated 24-h urinary sodium and potassium excretions from concentrations of these electrolytes in the baseline urine sample using the Kawasaki formulae [29]:

$$
\begin{aligned}
& \mathrm{eUNa}=22.99 \times 16.3 \times \sqrt{\left[\left(\frac{\mathrm{UNa}}{\mathrm{UCr}} \times 10\right) \times \mathrm{eUCr}\right]}, \\
& \mathrm{eUK}=39.10 \times 7.2 \times \sqrt{\left[\left(\frac{\mathrm{UK}}{\mathrm{UCr}} \times 10\right) \times \mathrm{eUCr}\right]} .
\end{aligned}
$$

and

In these equations, eUNa and eUK are the estimated 24-h sodium and potassium excretion in $\mathrm{mg}$ and in which UNa and UK are the urinary concentrations of sodium and potassium in $\mathrm{mmol} / \mathrm{l}$, respectively. To compare with international recommendations, and most of the previous studies, we transformed sodium and potassium from $\mathrm{mmol} / 24 \mathrm{~h}$ to $\mathrm{mg} / 24 \mathrm{~h}$ by using their molecular weight: 22.9 and 39.10, respectively.

$\mathrm{UCr}$ is the urinary concentration of creatinine in $\mathrm{mg} / \mathrm{dl}$ in the urine sample and eUCr is the estimated 24-h urinary creatinine excretion in $\mathrm{mg}$ calculated as followed:

$$
\begin{aligned}
& \mathrm{eUCr}=-12.63 \times \text { age }+15.12 \times \text { weight }+7.39 \\
& \quad \times \text { height }-79.9 \text { for men, and } \\
& \mathrm{eUCr}=-4.72 \times \text { age }+8.58 \times \text { weight }+5.09 \\
& \quad \times \text { height }-74.5 \text { for women }
\end{aligned}
$$

As eUNa/K can be directly defined as a function of $\mathrm{UNa} /$ UK measured in the sample after algebraic simplification (removing imprecision of the estimation of 24-h creatinine), our results apply also to measured $\mathrm{UNa} / \mathrm{UK}$ where eUNa/ $\mathrm{K}=C \times$ square root $(\mathrm{UNa} / \mathrm{UK})$ when $C=1.67$ for men. As expected, correlation between $\mathrm{eUNa} / \mathrm{K}$ and measured $\mathrm{UNa} / \mathrm{UK}$ was 1.0 (Spearman's correlation).

\section{Descriptive statistics}

Participants who lacked any of the baseline covariates or variables of interest $(n=703)$ or values at follow-up ( $n=1342$ ) were excluded from all statistical analyses that finally included 4141 participants. Distribution of continuous variables was graphically checked. To avoid extreme outliers, we decided to winsorize values of BMI, UCr, eUNa, eUK, urinary albumin and eGFR at percentiles 1 and 99\%.

Continuous variables were expressed in means and SDs except if the distribution was skewed, in which case variables were accordingly expressed in medians and interquartile ranges (IQR: 25th and 75th percentiles). Categorical variables were expressed in proportions. Continuous variables and categorical variables were compared by sex using the $t$ test - or the median test if the distribution was skewed - and the $\chi^{2}$ test for independence, respectively.

\section{Association analyses}

We graphically checked the distribution of the previously cited continuous variables and transformed some which were skewed: UCr was square-root transformed, ACR inversely square-root transformed and ultrasensitive CRP log transformed.

First, we performed univariate linear regressions describing $\triangle \mathrm{eGFR}$ as a function of age, sex, physical activity, smoking status, diabetes, antihypertensive medication, BMI, SBP, DBP, mean arterial BP (MAP), triglycerides, total cholesterol, uric acid, ultrasensitive CRP, 25D, 25D months tertiles, baseline eGFR, ACR, and eUNa, eUK or eUNa/K, separately. Variables with $P$ value 0.10 or less in univariate linear regressions were considered as significant and were chosen as covariates in multivariate linear regressions models as well as variables known to be associated in the literature with eGFR decline. We forced age and sex into the models. Other significant covariables were: physical activity, smoking status, diabetes, antihypertensive medication, SBP, BMI, triglycerides, uric acid, ultrasensitive CRP, 25D, ACR and baseline eGFR. eUNa and eUK or eUNa/K were the variables of interest. We chose SBP rather than MAP because of a greater adjusted $R$-squared value and a greater standardized coefficient of the absolute value. For the same reason, we chose 25D instead of month-specific terciles of the vitamin.

Then we generated three different multivariate linear regression models defining $\Delta$ eGFR (i.e. the outcome). Model 1 described $\Delta$ eGFR as a function of eUNa and eUK - or eUNa/K - adjusting for age and sex. Model 2 was adjusted for all previously described significant variables and ACR. Model 3 was similar to Model 2 but not adjusted for ACR. Models 1, 2 and 3 were either adjusted for baseline eGFR or not. Results are presented with the standardized regression coefficients $(\beta)$, calculated from standardized continuous variables (both dependant and independent), that is [variable $X$ - mean variable $X] / \mathrm{SD}$ of $X$, their $95 \%$ confidence intervals and the $P$ value of the variable of interest in the model from the Wald test.

Interactions between eUNa, eUK and eUNa/K with sex, age and hypertension status were tested by adding the interaction factor into Model 2: e.g hypertension*eUNa. An interaction with a $P$ value 0.10 or less was considered as significant. There were no significant interactions with sex, age or hypertension. We also tested interaction with hypertension status using eUNa, eUK and eUNa/K quintiles groups. We performed a likehood ratio test (LRT) to compare models with the interaction factor to the model without it, considering eUNa, eUK and eUNaK in quintiles groups instead of continuous variables: e.g hypertension*eUNa_quintiles. LRT $P$ values were more than 0.1 for all. 
TABLE 1. Participants' characteristics by sex (participants included in the follow-up, $n=4141$ )

\section{Variable}

Age (years)

Men, $n=1891$ (45.7\%)

Physically active, $n(\%)$

Active smoking, $n(\%)$

Diabetes, $n(\%)$

Antihypertensive medication, $n$ (\%)

BMl $\left(\mathrm{kg} / \mathrm{m}^{2}\right)$

Waist $(\mathrm{cm})$

SBP $(\mathrm{mmHg})$

$\mathrm{DBP}(\mathrm{mmHg})$

MAP (mmHg)

Serum cholesterol $(\mathrm{mmol} / \mathrm{l})$

Serum triglycerides $(\mathrm{mmol} / \mathrm{l})$

Serum uric acid (mg/dl)

Serum 25-hydroxyvitamin D (ng/ml)

hsCRP (mg/l)

eGFR $\left(\mathrm{ml} / \mathrm{min}\right.$ per $\left.1.73 \mathrm{~m}^{2}\right)$

$\triangle$ eGFR $\left(\mathrm{ml} / \mathrm{min}\right.$ per $1.73 \mathrm{~m}^{2}$ per year $)$

ACR $(\mathrm{mg} / \mathrm{g})$

eUNa (mg/24h)

eUK (mg/24h)

eUNa/K

50.4 [42.8-60.0]

$1259(67 \%)$

$516(27 \%)$

$157(8.3 \%)$

$343(18.1 \%)$

$26.4 \pm 3.8$

$95.2 \pm 10.9$

$131.3 \pm 16.3$

$81.1 \pm 10.7$

$97.9 \pm 11.8$

$5.59 \pm 1.03$

$1.3[0.9-1.9]$

$360 \pm 74$

$47.1 \pm 21.9$

$1.1[0.6-2.3]$

$87.7 \pm 14.7$

$-0.67 \pm 1.64$

4.3 [3.0-7.5]

$4262 \pm 1202$

$2557 \pm 543$

$1.71 \pm 0.51$
Women, $n=2250$ (54.3\%)

$\boldsymbol{P}$ value
52.5 [44.3-61.3]

$1522(68 \%)$

$72(3.2 \%)$

$303(13.5 \%)$

$24.8 \pm 4.6$

$82.5 \pm 12.1$

$123.6 \pm 17.6$

$76.9 \pm 10.4$

$95.5 \pm 11.9$

$5.60 \pm 1.00$

$1.0[0.7-1.3]$

$268 \pm 66$

$49.2 \pm 22.2$

$1.2[0.6-2.8]$

$84.0 \pm 14.6$

$-0.52 \pm 1.71$

5.5 [3.7-9.2]

$3502 \pm 1095$

$2246 \pm 486$

$1.59 \pm 0.50$
$<0.001$

0.037

$<0.001$

$<0.001$

$<0.001$

$<0.001$

$<0.001$

$<0.001$

$<0.001$

0.96

$<0.001$

$<0.001$

0.003

0.024

$<0.001$

0.007

$<0.001$

$<0.001$

$<0.001$

$<0.001$

Continuous variables are expressed as the mean \pm SD or median and IQR [25-75th percentiles] as appropriate. Categorical variables are expressed in numbers and percentages. ACR urinary albumin-to-creatinine ratio; CKD, chronic kidney disease; eGFR, estimated glomerular filtration rate using the CKD-Epidemiology Collaboration (CKD-EPI) equation; eUNa and eUK, estimated 24-h excretion of sodium and potassium, respectively; eUNa/K, ratio of eUNa to eUK; hsCRP, ultrasensitive C-reactive protein; MAP, mean arterial blood pressure.

To test the linearity as a condition for using linear regressions, we calculated $\Delta$ eGFR by quintiles of eUNa, eUK and $\mathrm{eUNa} / \mathrm{K}$ and we calculated $P$ for the trend for these variables. Linearity was present with all $P$ values less than 0.10 .

Main statistical analyses were performed using Stata 14.0 (StataCorp, College Station, Texas, USA).

\section{RESULTS}

We included 4141 participants without missing data and with a median follow-up of 5.4 (IQR: 5.3-5.6) years. Their characteristics by sex are shown in Table 1 . There were 54.3\% women. For the whole population, the median age was 51.5 (IQR: 43.6-60.6) years and mean baseline eGFR 85.7 (SD 14.8) $\mathrm{ml} / \mathrm{min}$ per $1.73 \mathrm{~m}^{2}$. Table 1 reported the mean eGFR (SD) by sex; the median eGFR [IQR] was 88.4 [77.7-98.6] and 83.9 [74.2-94.5] $\mathrm{ml} / \mathrm{min}$ per $1.73 \mathrm{~m}^{2}$ in men and women, respectively. The total mean eGFR decline in $\mathrm{ml} / \mathrm{min}$ per $1.73 \mathrm{~m}^{2}$ per year was -0.59 (SD 1.68) and the median -0.61 (IQR: $-1.58-0.45$ ). Men had higher prevalence of cardiovascular risk factors such as smoking, diabetes, antihypertensive medication, higher BMI, SBP, DBP, triglycerides and uric acid. eGFR at baseline was higher in men than in women but men showed a greater decline over time than women. Men had higher eUNa, eUK and eUNa/K than women. Women were older, had higher levels of vitamin D, higher CRP and albuminuria levels.

In Fig. 1 (as well as Supplementary Table S1, http:// links.lww.com/HJH/B89), we depicted unadjusted $\Delta$ eGFR by quintiles of eUNa, eUK and eUNa/K. This result shows an increase in eGFR decline with a significant trend across higher quintiles for the three variables of interest.

\section{Association analyses}

In univariate linear regressions, eUNa, eUK and eUNa/K were significantly associated with the decline in renal function, higher urinary levels of all parameters being associated with a steeper decrease in renal function. The multivariate linear regression models are presented in Table 2 adjusted or not for baseline eGFR. Models adjusted for baseline eGFR had a better predictive value when considering $R$-squared values. The addition of microalbuminuria had little impact on the coefficients. As shown in Table 2, we observed significant inverse association (=negative $\beta$-coefficients) for eUNa in both nonadjusted and adjusted models for baseline eGFR. To illustrate the size of the effect, an increment of $1 \mathrm{~g}$ of eUNa accelerates on average the kidney function decline by $0.1 \mathrm{ml} / \mathrm{min}$ per $1.73 \mathrm{~m}^{2}$ per year whenever adjusting for baseline eGFR. eUNa/K showed also significant negative $\beta$-coefficients in all models, meaning an inverse association with eGFR decline. Although eUK was also negatively associated with renal function decline in models not accounting for baseline eGFR, further adjustment for baseline eGFR changed the direction of the association, which became NS.

We did not found any interaction between age, sex or hypertension status and eUNa, eUK or eUNaK in our cohort neither using continuous nor categorical variables.

\section{Sensitive analyses}

We found similar results when we repeated the analyses after removing extreme outliers instead of winsorizing (p1, p99) variables.

To estimate the impact of a 'loss to follow-up bias', we compared characteristics between people included in the follow-up and those lost to follow-up (Supplementary Table S2, http://links.lww.com/HJH/B89). People included in the follow-up were younger and were healthier than people lost to follow-up: more physically active, less smokers, less diabetes, less antihypertensive treatment, lower BMI, lower SBP/DBP, lower triglycerides, lower uric acid, lower CRP, lower ACR and higher vitamin D values. There 
(a)

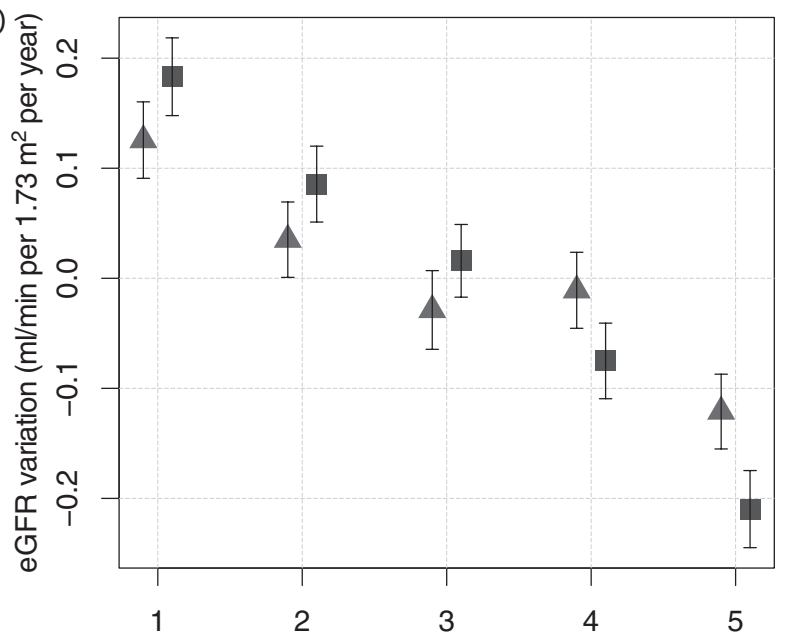

Quintiles of eUNa (squares) and eUK (triangles)

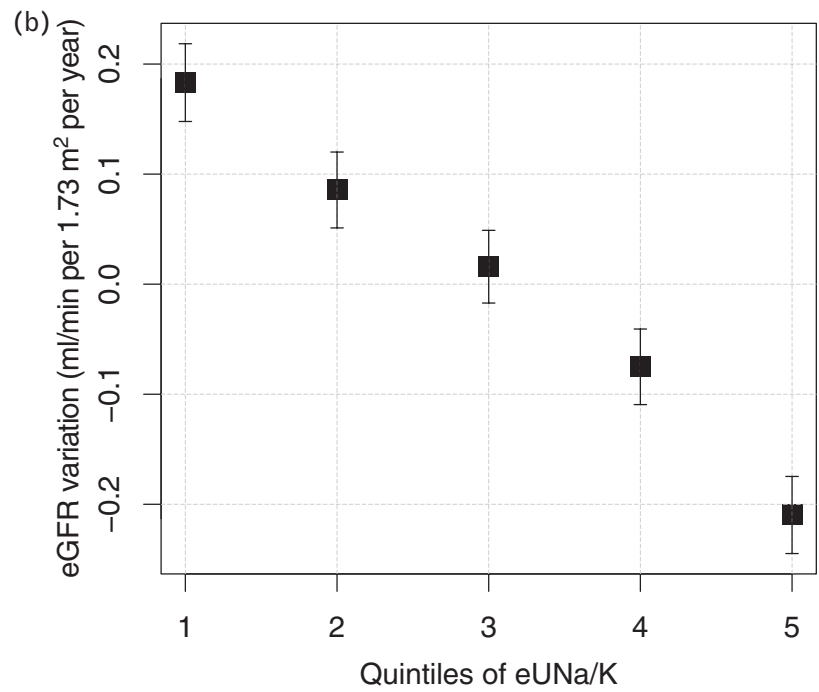

FIGURE 1 Yearly change in estimated glomerular filtration rate in $\mathrm{ml} / \mathrm{min}$ per $1.73 \mathrm{~m}^{2}$ per year (a) by quintiles of estimated 24-h excretion of sodium in $\mathrm{mg} /$ day (blue squares) and potassium in mg/day (red triangles); (b) by quintiles of estimated 24-h excretion sodium-to-potassium ratio $(n=4141)$. Vertical bars correspond to the standard errors of the mean. eGFR, estimated glomerular filtration rate; eUNa and eUK, estimated 24-h excretion of sodium and potassium, respectively; eUNa/K, ratio of eUNa to eUK. was no significant difference in baseline eGFR UNa, urinary concentration of potassium or UCr between these two groups. People included in follow-up had lower eUNa (and $\mathrm{eUNa} / \mathrm{K}$ ) than people lost to follow-up.

\section{DISGUSSION}

In this study, we found that high urinary sodium excretion and high urinary sodium-to-potassium excretion ratio were associated with a faster kidney function decline in adults from the general adult population, independently of major potential confounders such as baseline eGFR and albuminuria and BP. We could not find a significant protective or deleterious effect of urinary potassium excretion when accounting for baseline renal function. These results highlight the importance of dietary factors in kidney function decline and hence CKD prevention at a population level.

In some but not all observational studies, sodium intake, using 24-h urinary sodium excretion, has been associated with renal function decline and increased risk of CKD $[15,18]$. An increased risk of adverse cardiovascular outcome is also reported in patients with CKD when sodium intake is more than $4 \mathrm{~g}$ of sodium per day $[9,30]$, although it seems to be a J-shape curve association in the general population [13]. Moreover, there are limited experimental data studying the effect of lowering sodium intake on renal disease progression [31-34]. A hypothesis for the beneficial effect of lowering sodium intake is that, in combination with a low-protein diet and angiotensin-modulation therapy, there is a more pronounced decrease in intraglomerular pressure and proteinuria which could slow kidney disease progression [35-38]. In experimental models, sodium intake increases TGF- $\beta$ production and reactive oxygen species $[39,40]$. In addition to these effects, independent of BP, the adverse effect of sodium intake on vessels and hypertension could also explain the association with kidney disease progression [41,42].

We did not find any association between potassium excretion and renal function once adjusting for baseline renal function. Epidemiological studies have shown a protective effect of potassium intake on mortality and CV

TABLE 2. Multivariate regression analysis of estimated $24 \mathrm{~h}$ urinary excretion of sodium potassium and sodium-to-potassium ratio with the yearly change in estimated glomerular filtration rate $\left(\mathrm{ml} / \mathrm{min}\right.$ per $1.73 \mathrm{~m}^{2}$ per year) $(n=4141)$

\begin{tabular}{|c|c|c|c|c|c|c|}
\hline & \multicolumn{3}{|c|}{ Not adjusted for baseline eGFR } & \multicolumn{3}{|c|}{ Adjusted for baseline eGFR } \\
\hline & $\beta$ & $P$ value & $95 \% \mathrm{Cl}$ & $\beta$ & $P$ value & $95 \% \mathrm{Cl}$ \\
\hline \multicolumn{7}{|l|}{ Model 1} \\
\hline eUK & -0.03 & 0.17 & -0.06 to -0.01 & 0.03 & 0.33 & $-0.01-0.06$ \\
\hline eUNa/K & -0.07 & $<0.001$ & -0.10 to -0.04 & -0.06 & $<0.001$ & -0.08 to -0.03 \\
\hline \multicolumn{7}{|l|}{ Model 2} \\
\hline eUNa & -0.11 & $<0.001$ & -0.14 to -0.07 & -0.07 & $<0.001$ & -0.11 to -0.04 \\
\hline eUK & -0.05 & 0.008 & -0.08 to -0.01 & 0.02 & 0.30 & $-0.02-0.05$ \\
\hline $\mathrm{eUNa} / \mathrm{K}$ & -0.06 & $<0.001$ & -0.09 to -0.02 & -0.05 & $<0.001$ & -0.08 to -0.02 \\
\hline \multicolumn{7}{|l|}{ Model 3} \\
\hline eUNa & -0.11 & $<0.001$ & -0.14 to -0.07 & -0.07 & $<0.001$ & -0.11 to -0.04 \\
\hline eUK & -0.05 & 0.010 & -0.08 to -0.01 & 0.02 & 0.28 & $-0.01-0.05$ \\
\hline $\mathrm{eUNa} / \mathrm{K}$ & -0.05 & 0.001 & -0.08 to -0.02 & -0.05 & $<0.001$ & -0.08 to -0.02 \\
\hline
\end{tabular}

$\beta$-Coefficient of the regression line for each increase in 1SD of continuous dependent and independant variables. eUNa and eUK are the estimated $24-\mathrm{h}$ excretion of sodium and

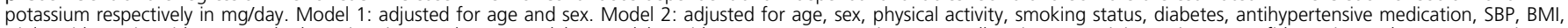
triglycerides, uric acid, CRP, 25-hydroxyvitamin D and ACR. Model 3: Model 2 without ACR as a covariate. ACR, albumin-to-creatinine ratio; CI, confidence interval; CRP, C-reactive protein; eGFR, estimated glomerular filtration rate; eUK, estimated 24-h urinary potassium excretion; eUNa, estimated 24-h urinary sodium excretion; eUNa/K, ratio of eUNa to eUK. 
disease $[12,19,43]$. The probable different effects of sodium and potassium on kidney function decline can be explained by their opposite cellular effects involving, for example, opposite action on nitric oxide release. Potassium also seems to have a direct effect on sodium excretion in animals by controlling the phosphorylation of sodium-chloride symporter $[5,44]$. However, data are conflicting: a recent study found higher potassium intake to be associated with kidney disease progression in CKD patients and another one described a higher risk of developing CKD with low potassium [15,19]. Despite these conflicting results, the WHO has reiterated the recommendation of reducing sodium intake and increasing potassium intake [43].

We have shown that a higher $\mathrm{Na} / \mathrm{K}$ ratio was associated with a steeper decline in renal function. We only have found one study in a general population from Japan analysing longitudinally $\mathrm{Na} / \mathrm{K}$ ratio as a predictor of renal function [45]. Similar to our study, the authors described that higher baseline ratio was associated with greater eGFR decline. However, their analyses mainly focused on BP. Even the PREVEND study, a population-based study without CKD, had not looked at the urinary ratio [19]. A higher ratio may reflect a higher sodium intake, a lower potassium intake or both. The $\mathrm{Na} / \mathrm{K}$ ratio is robust to errors related to incomplete collections and is independent of creatinine measurements [23]. $\mathrm{Na} / \mathrm{K}$ ratio has been studied in relation to BP and mortality and higher ratios were associated with a higher BP and higher risk of hypertension in the general population and were predictive of worse outcomes [46,47]. The estimated reduction was even larger when using the ratio compared with sodium and potassium excretions separately. More recent studies also confirm these results [5,48,49]. A recent study looked at the effects of the Dietary Approaches to Stop Hypertension (DASH) diet - thus consequently with a lower $\mathrm{Na} / \mathrm{K}$ ratio - on CKD incidence. In this study, volunteers in the highest tertile of DASH score (DASH-style diet) were 16\% less likely to develop CKD comparing with those in the lowest tertile DASH score [50].

Our study has several strengths. First, it has an optimal design, being observational and population-based with a long period between baseline and follow-up investigations. Second, we used Kawasaki formulae to estimate 24-h excretions of sodium and potassium - which are more precise than simple urine sample concentrations - as a marker of sodium and potassium intakes. Third, we also adjusted models for baseline eGFR and for several risk factors for renal function decline.

There are, however, some limitations. We measured urinary sodium and potassium in a single spot urine sample at baseline. Thus, we neglected individual variations in excretion of these electrolytes related to circadian rhythms and dietary variations. Moreover, we used Kawasaki formulae which did not seem to match exactly with 24-h urines in a recent study [51]. On the other hand, the use of $\mathrm{Na} / \mathrm{K}$ ratio overcomes the problem of the sample variability and the lack of precision from formulae used to extrapolate 24-h excretion. Moreover, the correlation between spot and 24-h urine was better for $\mathrm{Na} / \mathrm{K}$ ratio than for sodium or potassium, taken alone [25]. Residual confounding is still possible. Finally, regarding the outcome, we estimated the decline using two creatinine measures at a 5-year interval.
With such two time-points data, we assumed linearity of the renal function decline and did not account for variability in the creatinine values. A recent article suggested that in most cases assuming linearity of renal function decline is correct [52]. Of course, with only two measures, we cannot exclude that we had overestimated or underestimated the decline in eGFR, by including participants with acute renal disease at baseline or follow-up.

In conclusion, we observed a significant linear association between urinary sodium excretion and age-related kidney function decline. We found no significant association between urinary potassium excretion and age-related kidney function decline when taking into account baseline renal function. $\mathrm{Na} / \mathrm{K}$ ratio was also a predictor of agerelated kidney function decline but essentially driven by the sodium component of the ratio as it was less predictive than sodium alone. Our results suggest that reducing sodium intake and having a low-dietary $\mathrm{Na} / \mathrm{K}$ ratio may slow down the progression to CKD. However, whether a lower $\mathrm{Na} / \mathrm{K}$ ratio should be obtained by reducing sodium intake only, both reducing sodium and increasing potassium intakes or increasing potassium intake alone is not known. This may well depend on the level of eGFR at which the intervention is introduced. In addition, whether nutritional interventions, such as low-sodium and highpotassium diet, may help preventing CKD needs further exploration.

\section{AGKNOWLEDGEMENTS}

The CoLaus study was supported by research grants from GlaxoSmithKline, the Faculty of Biology and Medicine of Lausanne, Switzerland, and the Swiss National Science Foundation (grant nos.: 33CSCO-122661 and 33CSCO139468). BP was partly funded by the Swiss National Foundation (grant MHV no. PMPDP3-171352). The funders had no role in study design, data collection and analysis, decision to publish or preparation of the article. We also thank Nadine Nägele for measuring urinary electrolytes.

Previous presentations: Abstract presented during the 55th ERA-EDTA Congress in Copenhagen in May 2018 as a poster and during the Swiss Society of General Internal Medicine (SSMI) Congress in Basel in May 2018 as an oral presentation.

\section{Conflicts of interest}

There are no conflicts of interest.

\section{REFERENGES}

1. Mente A, O'Donnell MJ, Rangarajan S, McQueen MJ, Poirier P, Wielgosz A, et al. Association of urinary sodium and potassium excretion with blood pressure. N Engl J Med 2014; 371:601-611.

2. Elliott P, Stamler J, Nichols R, Dyer AR, Stamler R, Kesteloot H, et al. Intersalt revisited: further analyses of 24 hour sodium excretion and blood pressure within and across populations. Intersalt Cooperative Research Group. BMJ 1996; 312:1249-1253.

3. O'Donnell M, Mente A, Rangarajan S, McQueen MJ, Wang X, Liu L, et al. Urinary sodium and potassium excretion, mortality, and cardiovascular events. N Engl J Med 2014; 371:612-623.

4. Aburto NJ, Hanson S, Gutierrez H, Hooper L, Elliott P, Cappuccio FP. Effect of increased potassium intake on cardiovascular risk factors and disease: systematic review and meta-analyses. BMJ 2013; 346:f1378. 
5. Yang Q, Liu T, Kuklina EV, Flanders WD, Hong Y, Gillespie C, et al. Sodium and potassium intake and mortality among US adults: prospective data from the Third National Health and Nutrition Examination Survey. Arch Intern Med 2011; 171:1183-1191.

6. Cook NR, Obarzanek E, Cutler JA, Buring JE, Rexrode KM, Kumanyika SK, et al. Joint effects of sodium and potassium intake on subsequent cardiovascular disease: the Trials of Hypertension Prevention followup study. Arch Intern Med 2009; 169:32-40.

7. Stolarz-Skrzypek K. Fatal and nonfatal outcomes, incidence of hypertension, and blood pressure changes in relation to urinary sodium excretion. JAMA 2011; 305:1777.

8. Mente A, O'Donnell M, Rangarajan S, McQueen M, Dagenais G, Wielgosz A, et al. Urinary sodium excretion, blood pressure, cardiovascular disease, and mortality: a community-level prospective epidemiological cohort study. Lancet 2018; 392:496-506.

9. He J, Mills KT, Appel LJ, Yang W, Chen J, Lee BT, et al. Urinary sodium and potassium excretion and CKD progression. J Am Soc Nephrol 2016; 27:1202-1212.

10. Fan L, Tighiouart H, Levey AS, Beck GJ, Sarnak MJ. Urinary sodium excretion and kidney failure in nondiabetic chronic kidney disease. Kidney Int 2014; 86:582-588.

11. Nagata T, Sobajima H, Ohashi N, Hirakawa A, Katsuno T, Yasuda Y, et al. Association between $24 \mathrm{~h}$ urinary sodium and potassium excretion and estimated glomerular filtration rate (eGFR) decline or death in patients with diabetes mellitus and eGFR more than $30 \mathrm{ml} / \mathrm{min} / 1.73 \mathrm{~m}^{2}$. PLoS One 2016; 11:e152306.

12. Smyth A, Dunkler D, Gao P, Teo KK, Yusuf S, O'Donnell MJ, et al. The relationship between estimated sodium and potassium excretion and subsequent renal outcomes. Kidney Int 2014; 86:1205-1212.

13. Kieneker LM, Bakker SJL, de Boer RA, Navis GJ, Gansevoort RT, Joosten MM. Low potassium excretion but not high sodium excretion is associated with increased risk of developing chronic kidney disease. Kidney Int 2016; 90:888-896.

14. Dunkler D, Dehghan M, Teo KK, Heinze G, Gao P, Kohl M, et al. Diet and kidney disease in high-risk individuals with type 2 diabetes mellitus. JAMA Intern Med 2013; 173:1682-1692.

15. Lin J, Hu FB, Curhan GC. Associations of diet with albuminuria and kidney function decline. Clin J Am Soc Nephrol 2010; 5:836-843.

16. Sharma S, McFann K, Chonchol M, de Boer IH, Kendrick J. Association between dietary sodium and potassium intake with chronic kidney disease in US adults: a cross-sectional study. Am J Nephrol 2013; 37:526-533.

17. Sanders PW. Effect of salt intake on progression of chronic kidney disease. Curr Opin Nephrol Hypertens 2006; 15:54-60.

18. Fukui M, Tanaka M, Toda H, Asano M, Yamazaki M, Hasegawa G, et al. Low serum potassium concentration is a predictor of chronic kidney disease. Int J Clin Pract 2014; 68:700-704.

19. Araki S, Haneda M, Koya D, Kondo K, Tanaka S, Arima H, et al. Urinary potassium excretion and renal and cardiovascular complications in patients with type 2 diabetes and normal renal function. Clin J Am Soc Nephrol 2015; 10:2152-2158.

20. Ko BJ, Chang Y, Ryu S, Kim EM, Lee MY, Hyun YY, et al. Dietary acid load and chronic kidney disease in elderly adults: protein and potassium intake. PLoS One 2017; 12:e0185069.

21. Oberleithner H, Callies C, Kusche-Vihrog K, Schillers H, Shahin V, Riethmüller $\mathrm{C}$, et al. Potassium softens vascular endothelium and increases nitric oxide release. Proc Natl Acad Sci U S A 2009; 106: 2829-2834

22. Fang Y, Mu JJ, He LC, Wang SC, Liu ZQ. Salt loading on plasma asymmetrical dimethylarginine and the protective role of potassium supplement in normotensive salt-sensitive asians. Hypertension 2006; 48:724-729.

23. Iwahori T, Miura K, Ueshima H. Time to consider use of the sodium-topotassium ratio for practical sodium reduction and potassium increase. Nutrients 2017; 700:1-11; doi:10.3390/nu9070700.

24. Yatabe MS, Iwahori T, Watanabe A, Takano K, Sanada H, Watanabe T, et al. Urinary sodium-to-potassium ratio tracks the changes in salt intake during an experimental feeding study using standardized lowsalt and high-salt meals among healthy Japanese volunteers. Nutrients 2017; 951:1-13; doi:10.3390/nu9090951

25. Iwahori T, Miura K, Ueshima $\mathrm{H}$, Chan $\mathrm{Q}$, Dyer AR, Elliott $\mathrm{P}$, et al. Estimating 24-h urinary sodium/potassium ratio from casual ('spot') urinary sodium/potassium ratio: the INTERSALT Study. Int J Epidemiol 2017; 46:1564-1572.
26. Adrogué HJ, Madias NE. Sodium surfeit and potassium deficit: keys to the pathogenesis of hypertension. J Am Soc Hypertens 2014; 8:203-213.

27. Firmann M, Mayor V, Vidal PM, Bochud M, Pécoud A, Hayoz D, et al. The CoLaus study: a population-based study to investigate the epidemiology and genetic determinants of cardiovascular risk factors and metabolic syndrome. BMC Cardiovasc Disord 2008; 8:6.

28. Levey AS, Stevens LA, Schmid CH, Zhang YL, Castro AF, Feldman HI, et al. A new equation to estimate glomerular filtration rate. Ann Intern Med 2009; 150:604-612.

29. Kawasaki T, Itoh K, Uezono K, Sasaki H. A simple method for estimating $24 \mathrm{~h}$ urinary sodium and potassium excretion from second morning voiding urine specimen in adults. Clin Exp Pharmacol Physiol 1993; 20:7-14

30. Mills KT, Chen J, Yang W, Appel LJ, Kusek JW, Alper A, et al. Sodium excretion and the risk of cardiovascular disease in patients with chronic kidney disease. JAMA 2016; 315:2200.

31. O'Donnell M, Mente A, Yusuf S. Sodium intake and cardiovascular health. Circ Res 2015; 116:1046-1057.

32. Kwakernaak AJ, Krikken JA, Binnenmars SH, Visser FW, Hemmelder $\mathrm{MH}$, Woittiez AJ, et al. Effects of sodium restriction and hydrochlorothiazide on RAAS blockade efficacy in diabetic nephropathy: a randomised clinical trial. Lancet Diabetes Endocrinol 2014; 2:385-395.

33. McMahon EJ, Bauer JD, Hawley CM, Isbel NM, Stowasser M, Johnson DW, et al. A randomized trial of dietary sodium restriction in CKD. $J \mathrm{Am}$ Soc Nephrol 2013; 24:2096-2103.

34. Swift PA, Markandu ND, Sagnella GA, He FJ, MacGregor GA. Modest salt reduction reduces blood pressure and urine protein excretion in black hypertensives: a randomized control trial. Hypertension 2005; 46:308-312

35. Kalantar-Zadeh K, Fouque D. Nutritional management of chronic kidney disease. $N$ Engl J Med 2017; 377:1765-1776.

36. Vegter S, Perna A, Postma MJ, Navis G, Remuzzi G, Ruggenenti P. Sodium intake, ACE inhibition, and progression to ESRD. J Am Soc Nephrol 2012; 23:165-173.

37. Heerspink HJL, Holtkamp FA, Parving HH, Navis GJ, Lewis JB, Ritz E, et al. Moderation of dietary sodium potentiates the renal and cardiovascular protective effects of angiotensin receptor blockers. Kidney Int 2012; 82:330-337.

38. Weir MR, Fink JC. Salt intake and progression of chronic kidney disease: an overlooked modifiable exposure? A commentary. Am J Kidney Dis 2005; 45:176-188.

39. Kitiyakara C, Chabrashvili T, Chen Y, Blau J, Karber A, Aslam S, et al. Salt intake, oxidative stress, and renal expression of NADPH oxidase and superoxide dismutase. J Am Soc Nephrol 2003; 14:2775-2782.

40. Ying WZ, Sanders PW. Dietary salt increases endothelial nitric oxide synthase and TGF-beta1 in rat aortic endothelium. Am J Physiol 1999; 277:H1293-H1298.

41. Morrison AC, Ness RB. Sodium intake and cardiovascular disease. Annu Rev Public Health 2011; 32:71-90.

42. Sanders PW. Vascular consequences of dietary salt intake. Am J Physiol Renal Physiol 2009; 297:F237-F243.

43. World Health Organization. Guideline: potassium intake for adults and children. Geneva: World Health Organization; 2012; http:// www.ncbi.nlm.nih.gov/books/NBK132470/. [Accessed 23 November 2017].

44. McDonough AA, Veiras LC, Guevara CA, Ralph DL. Cardiovascular benefits associated with higher dietary $\mathrm{K}^{+}$vs. lower dietary $\mathrm{Na}^{+}$: evidence from population and mechanistic studies. Am J Physiol Endocrinol Metab 2017; 312:E348-E356.

45. Tabara Y, Takahashi Y, Setoh K, Kawaguchi T, Kosugi S, Nakayama T, et al. Prognostic significance of spot urine $\mathrm{Na} / \mathrm{K}$ for longitudinal changes in blood pressure and renal function: the Nagahama study. Am J Hypertens 2017; 30:899-906.

46. [No authors listed]. Intersalt: an international study of electrolyte excretion and blood pressure. Results for 24 hour urinary sodium and potassium excretion. Intersalt Cooperative Research Group. BMJ 1988; 297:319-328.

47. Stamler J, Rose G, Stamler R, Elliott P, Dyer A, Marmot M. INTERSALT study findings. Public health and medical care implications. Hypertension 1989; 14:570-577.

48. Okayama A, Okuda N, Miura K, Okamura T, Hayakawa T, Akasaka H, et al. Dietary sodium-to-potassium ratio as a risk factor for stroke, cardiovascular disease and all-cause mortality in Japan: the NIPPON DATA80 cohort study. BMJ Open 2016; 6:e011632. 
49. Willey J, Gardener H, Cespedes S, Cheung YK, Sacco RL, Elkind MSV. Dietary sodium to potassium ratio and risk of stroke in a multiethnic urban population: the Northern Manhattan Study. Stroke 2017; 48:2979-2983.

50. Rebholz CM, Crews DC, Grams ME, Steffen LM, Levey AS, Miller ER, et al. DASH (Dietary Approaches to Stop Hypertension) diet and risk of subsequent kidney disease. Am J Kidney Dis 2016; 68:853-861.
51. Polonia J, Lobo MF, Martins L, Pinto F, Nazare J. Estimation of populational 24-h urinary sodium and potassium excretion from spot urine samples: evaluation of four formulas in a large national representative population. J Hypertens 2017; 35:477-486.

52. Weldegiorgis M, de Zeeuw D, Li L, Parving HH, Hou FF, Remuzzi G, et al. Longitudinal estimated GFR trajectories in patients with and without type 2 diabetes and nephropathy. Am J Kidney Dis 2018; $71: 91-101$. 